\title{
One-loop renormalization of Lorentz-violating electrodynamics
}

\author{
V. Alan Kostelecký \\ Physics Department, Indiana University, Bloomington, Indiana 47405 \\ Charles D. Lane \\ Physics Department, Berry College, Mount Berry, Georgia 30149 \\ Austin G. M. Pickering \\ Physics Department, Indiana University, Bloomington, Indiana 47405
}

(Received 10 October 2001; published 31 January 2002)

\begin{abstract}
We show that the general Lorentz- and CPT-violating extension of quantum electrodynamics is one-loop renormalizable. The one-loop Lorentz-violating beta functions are obtained, and the running of the coefficients for Lorentz and $C P T$ violation is determined. Some implications for theory and experiment are discussed.
\end{abstract}

DOI: 10.1103/PhysRevD.65.056006

PACS number(s): 11.30.Cp, 11.10.Hi, 12.20.-m

\section{INTRODUCTION}

The standard model of particle physics is invariant under Lorentz and CPT transformations. However, the possibility that nature exhibits small violations of Lorentz and $C P T$ symmetry appears compatible with quantum field theory and with existing experiments [1]. A general description of the associated effects can be formulated at the level of quantum field theory as a Lorentz- and CPT-violating standard-model extension [2]. The Lagrangian of this theory includes all possible operators that are observer Lorentz scalars and that are formed from standard-model fields and coupling coefficients with Lorentz indices. Imposing the usual $\mathrm{SU}(3) \times \mathrm{SU}(2)$ $\times \mathrm{U}(1)$ gauge invariance and restricting attention to lowenergy effects, the standard-model extension is well approximated by the usual standard model together with all possible Lorentz-violating terms of mass dimension four or less that are constructed from standard-model fields.

Among the interesting open issues associated with Lorentz and CPT violation is the manner in which the lowenergy theory connects to the underlying Planck-level theory as the energy scale is increased. Some insight into this link has been obtained through the study of causality and stability in Lorentz-violating quantum field theory [3]. In the present work, we study a different facet of this connection, involving the role of radiative corrections and the renormalization group.

To provide a definite focus and a tractable scope, we limit attention here to the special case of effects from one-loop divergences in the Lorentz- and $C P T$-violating quantum electrodynamics (QED) of a single fermion. This QED extension can be regarded as a specific limit of the standardmodel extension. Even in this simplified limit, relatively little is known about loop effects. Some one-loop calculations have been performed in the photon sector $[2,4]$, but a comprehensive treatment has been lacking. One goal of the present work is to fill this gap. Tools such as a generalization of the Furry theorem [5] are developed, and all divergent one-loop corrections are determined. We use these to prove one-loop renormalizability and gauge invariance of the theory. The calculations are presented here in dimensional regularization [6,7], although we have also checked their validity in Pauli-Villars regularization [8].

In the standard-model extension, all Lorentz- and $C P T$-violating effects are controlled by a set of coefficients that can be regarded as originating in an underlying theory at the Planck scale. For example, they might be associated with expectation values in string theory [9], and specific nonzero coefficients emerge in realistic noncommutative field theories [10]. Several of these coefficients in different sectors of the standard-model extension are now bounded by experiments involving hadrons [11-14], protons and neutrons [15], electrons [16,17], photons [18], and muons [19]. Our results in this work can be used to gain insight into the relationships among coefficients for Lorentz and $C P T$ violation as the scale ranges between low and high energies.

A basic tool for studying quantum physics over different scales is the renormalization group [20,21]. Here, we discuss its relevance in the context of the Lorentz- and $C P T$-violating standard-model extension. We use our calculations of the one-loop divergences to extract the corresponding beta functions for all the coefficients for Lorentz and $C P T$ violation in the general QED extension. Solving the associated set of coupled partial-differential equations for the renormalized coefficients yields their running as the scale is changed. Knowledge of this running offers some insight into the possible relative sizes of nonzero Lorentz- and CPT-violating effects.

This paper is organized as follows. Section II provides some basic information about the general Lorentz- and $C P T$-violating QED extension. Renormalizability of the theory is considered in Sec. III. Some general issues are discussed, following which we present the results of our oneloop calculation. We establish the absence of divergent cubic and quartic photon interactions, present explicit results for all divergent radiative corrections to the Lagrangian, and show that the Ward identities are preserved at this order. Section IV begins with a discussion of the application of the renormalization-group method in the context of Lorentz and $C P T$ violation. The one-loop beta functions for all parameters in the theory are then derived. The resulting coupled partial-differential equations are solved for the running pa- 
rameters, and some implications for experiment are considered. A summary is provided in Sec. V. The Feynman rules used in our analysis are presented in the Appendix. Throughout this work, our notation is that of Refs. [2,3].

\section{BASICS}

The Lagrangian $\mathcal{L}$ of the general Lorentz- and $C P T$-violating QED extension for a fermion field $\psi$ of mass $m$ in four spacetime dimensions can be written as [2]

$$
\begin{aligned}
\mathcal{L}= & \frac{1}{2} i \bar{\psi} \Gamma^{\mu} \overleftrightarrow{D}_{\mu} \psi-\bar{\psi} M \psi-\frac{1}{4} F^{\mu \nu} F_{\mu \nu} \\
& -\frac{1}{4}\left(k_{F}\right)_{\kappa \lambda \mu \nu} F^{\kappa \lambda} F^{\mu \nu}+\frac{1}{2}\left(k_{A F}\right)^{\kappa} \epsilon_{\kappa \lambda \mu \nu} A^{\lambda} F^{\mu \nu},
\end{aligned}
$$

where $\Gamma^{\nu}=\gamma^{\nu}+\Gamma_{1}^{\nu}$ and $M=m+M_{1}$, with

$$
\begin{aligned}
\Gamma_{1}^{\nu} & \equiv c^{\mu \nu} \gamma_{\mu}+d^{\mu \nu} \gamma_{5} \gamma_{\mu}+e^{\nu}+i f^{\nu} \gamma_{5}+\frac{1}{2} g^{\lambda \mu \nu} \sigma_{\lambda \mu}, \\
M_{1} & \equiv a_{\mu} \gamma^{\mu}+b_{\mu} \gamma_{5} \gamma^{\mu}+\frac{1}{2} H_{\mu \nu} \sigma^{\mu \nu} .
\end{aligned}
$$

As usual, we define the covariant derivative $D_{\mu} \equiv \partial_{\mu}$ $+i q A_{\mu}$ and the electromagnetic field strength $F_{\mu \nu} \equiv \partial_{\mu} A_{\nu}$ $-\partial_{\nu} A_{\mu}$.

In the fermion sector, the coefficients for Lorentz violation are $a_{\mu}, b_{\mu}, c_{\mu \nu}, d_{\mu \nu}, e_{\mu}, f_{\mu}, g_{\lambda \mu \nu}, H_{\mu \nu}$. Of these, $a_{\mu}, b_{\mu}, e_{\mu}, f_{\mu}, g_{\lambda \mu \nu}$ govern $C P T$ violation. The coefficients $a_{\mu}, b_{\mu}, H_{\mu \nu}$ have dimensions of mass, while $c_{\mu \nu}$, $d_{\mu \nu}, e_{\mu}, f_{\mu}, g_{\lambda \mu \nu}$ are dimensionless. Both $c_{\mu \nu}$ and $d_{\mu \nu}$ can be taken to be traceless, while $H_{\mu \nu}$ is antisymmetric and $g_{\lambda \mu \nu}$ is antisymmetric on its first two indices. In the photon sector, the coefficients for Lorentz violation are $\left(k_{A F}\right)_{\mu}$, $\left(k_{F}\right)_{\kappa \lambda \mu \nu}$. CPT violation is governed only by $\left(k_{A F}\right)_{\mu}$, which has dimensions of mass. The coefficient $\left(k_{F}\right)_{\kappa \lambda \mu \nu}$ is dimensionless, has the symmetry properties of the Riemann tensor, and is double traceless:

$$
\begin{aligned}
\left(k_{F}\right)_{\kappa \lambda \mu \nu}=\left(k_{F}\right)_{\mu \nu \kappa \lambda} & =-\left(k_{F}\right)_{\lambda \kappa \mu \nu}, \\
\left(k_{F}\right)_{\kappa \lambda \mu \nu}+\left(k_{F}\right)_{\kappa \mu \nu \lambda}+\left(k_{F}\right)_{\kappa \nu \lambda \mu} & =0, \\
\left(k_{F}\right)_{\mu \nu}{ }^{\mu \nu} & =0 .
\end{aligned}
$$

The requirement that the Lagrangian be Hermitian implies that all the coefficients for Lorentz violation are real.

In the Lorentz-violating theory (1), two distinct types of Lorentz transformation are relevant [2]. The Lagrangian (1) is invariant under observer Lorentz transformations: rotations and boosts of the observer inertial frame have no effect on the physics because both the field operators and the coefficients for Lorentz violation transform covariantly and because each term in the Lagrangian (1) is an observer scalar. These coordinate transformations are distinct from rotations and boosts of a particle or localized field configuration within a fixed observer inertial frame. The latter are called particle Lorentz transformations. They leave unchanged the
TABLE I. Discrete-symmetry properties.

\begin{tabular}{lccccccc}
\hline \hline & $C$ & $P$ & $T$ & $C P$ & $C T$ & $P T$ & $C P T$ \\
\hline$c_{00},\left(k_{F}\right)_{0 j 0 k}$, & & & & & & & \\
$c_{j k},\left(k_{F}\right)_{j k l m}$ & + & + & + & + & + & + & + \\
$b_{j}, g_{j 0 l}, g_{j k 0},\left(k_{A F}\right)_{j}$ & + & + & - & + & - & - & - \\
$b_{0}, g_{j 00}, g_{j k l},\left(k_{A F}\right)_{0}$ & + & - & + & - & + & - & - \\
$c_{0 j}, c_{j 0},\left(k_{F}\right)_{0 j k l}$ & + & - & - & - & - & + & + \\
$a_{0}, e_{0}, f_{j}$ & - & + & + & - & - & + & - \\
$H_{j k}, d_{0 j}, d_{j 0}$ & - & + & - & - & + & - & + \\
$H_{0 j}, d_{00}, d_{j k}$ & - & - & + & + & - & - & + \\
$a_{j}, e_{j}, f_{0}$ & - & - & - & + & + & + & - \\
\hline
\end{tabular}

coefficients for Lorentz violation and hence change the physics. The theory (1) therefore violates particle Lorentz invariance.

Since the coefficients for Lorentz violation are transformed by an observer Lorentz transformation, an appropriate boost can make at least some of them large. To avoid issues with perturbation theory, in this work we limit calculations to concordant frames [3]: ones in which the coefficients for Lorentz violation are small compared to the fermion mass $m$ or to the dimensionless charge $q$. Any frame in which the Earth moves nonrelativistically is known experimentally to be concordant, so this restriction offers no practical difficulty in applying our results. However, to maintain generality, we make no assumptions concerning the relative sizes of the different coefficients for Lorentz violation.

The hierarchy of scales between the coefficients for Lorentz violation and the parameters $m, q$ has implications for the structure of dominant one-loop Lorentz- and $C P T$-violating effects. In particular, since Lorentz and $C P T$ violation can be assumed small and since we are interested in leading-order Lorentz- and $C P T$-violating effects, it suffices for the purposes of this work to define a one-loop diagram as one that contains exactly one closed loop and is either zeroth or first order in coefficients for Lorentz violation. All relevant one-loop diagrams are therefore $\mathcal{O}\left(q^{2}\right)$ and at most linear in the coefficients for Lorentz violation. Note that it would be invalid to include nonlinear contributions from the coefficients for Lorentz violation without also considering multiloop contributions at high order in $q$, which could be the same order of magnitude.

Combined with symmetry arguments, the restriction to linear Lorentz- and CPT-violating effects enables some strong predictions about which terms in the Lagrangian (1) can contribute to the renormalization of any given coefficient. Since QED preserves $C, P$, and $T$ invariance, any Lorentz-violating terms mixing linearly under radiative corrections must have identical $C, P$, and $T$ transformation properties. Table I lists these properties for the field operators appearing in the Lagrangian (1). For brevity, the corresponding coefficients for Lorentz violation are listed in the table rather than the field operators themselves.

The table reveals terms for which the $C, P, T$ symmetries allow mixing under renormalization group flow. Other restrictions also exist. Since in what follows we adopt a massindependent renormalization scheme, operators associated 


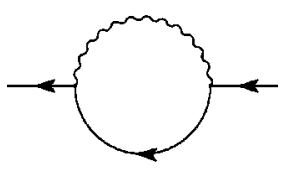

(a)

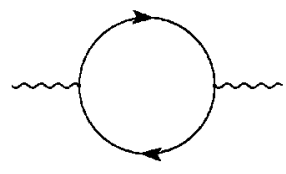

(b)

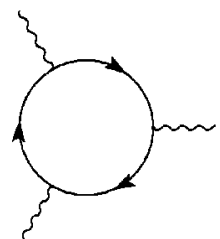

(c)

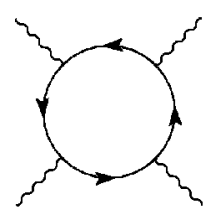

(d)

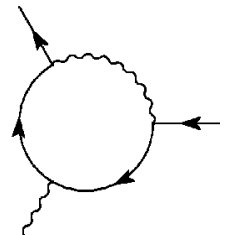

(e)
FIG. 1. One-loop topologies for QED. with dimensionless coefficients cannot receive corrections from ones associated with massive coefficients. Thus, for example, $a_{\mu}$ can receive corrections from $e_{\mu}$ on symmetry grounds, but $e_{\mu}$ cannot receive corrections from $a_{\mu}$ on dimensional grounds. There are also restrictions arising from the rotational invariance of QED. For instance, rotational symmetry prevents $e_{0}$ and $f_{j}$ from mixing at this level of approximation, even though this would be allowed by the $C, \quad P, T$ properties of the corresponding field operators. All these features are confirmed by the explicit calculations that follow.

\section{RENORMALIZABILITY AT ONE LOOP}

In this section, we give an explicit demonstration of renormalizability at one loop for the QED extension (1). Following some general considerations, we obtain a generalization of the Furry theorem and establish the finiteness of the photon vertices. The divergent propagator and vertex corrections are given along with the renormalization factors, and the Ward identities are shown to hold.

\section{A. Setup}

Renormalizability of a quantum field theory can be viewed as the requirements that the number of primitively divergent one-particle-irreducible (1PI) diagrams is finite and that the number of parameters suffices to absorb the corresponding infinities. To establish renormalizability of the QED extension (1), we first determine the superficial degree of divergence of a general Feynman diagram. Using the Feynman rules for the theory provided in the Appendix, it follows that the superficial degree of divergence $D$ of a general diagram in the QED extension is

$$
D=4-\frac{3}{2} E_{\psi}-E_{A}-V_{M_{1}}-V_{A F},
$$

where $E_{\psi}$ is the number of external fermion legs, $E_{A}$ is the number of external photon legs, $V_{M_{1}}$ is the number of insertions of the $M_{1}$ operator in a fermion propagator, and $V_{A F}$ is the number of insertions of $\left(k_{A F}\right)_{\mu}$ in a photon propagator.

The expression (4) shows that there are a finite number of potentially divergent 1PI diagrams at one loop. Their topologies correspond to those of the divergent diagrams associated with conventional QED, displayed in Fig. 1. However, in addition to these usual diagrams, there is a set of diagrams obtained from them by single insertions of coefficients for Lorentz violation allowed by the Feynman rules. All such insertions lead to 1PI divergent diagrams, except for those involving the coefficients $a_{\mu}, b_{\mu}, H_{\mu \nu},\left(k_{A F}\right)_{\mu}$ inserted into logarithmically divergent diagrams of conventional QED.
These exceptions arise because the mass dimensionality of the coefficients involved ensures a finite result.

As an illustration, the QED diagram in Fig. 1(e) leads to the set of divergent contributions illustrated in Fig. 2. In these diagrams, dimensionless coefficients for Lorentz violation are represented as filled circles while the others are represented by crosses. The notation is detailed in the Appendix.

As usual, each additional 1PI diagram involves a one-loop integration. To evaluate the divergent contributions of these diagrams to the effective action, a regularization scheme for the loop integrations is needed. In this paper, we adopt dimensional regularization. However, we have also repeated our calculations using Pauli-Villars regularization. It turns out that the usual correspondence between the two schemes holds, supporting the expected scheme independence of the physical results.

In dimensional regularization, the presence of particle Lorentz violation has little effect on the standard evaluation of loop integrals. Although use is sometimes made of the Lorentz properties of the integrand, the standard techniques hold because the integrands involve momentum variables that behave covariantly under both observer and particle transformations, as usual. Moreover, the linearity of Lorentz violation means that the role of the coefficients for Lorentz violation is limited in this context to contraction with the result of the integration. For similar reasons, no new issues arise with manipulations such as Wick rotations. We can therefore perform the necessary regularization of divergent

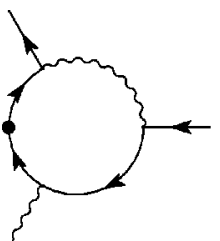

(a)

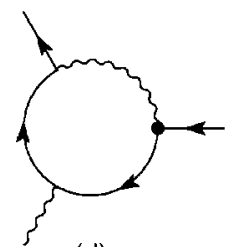

(d)

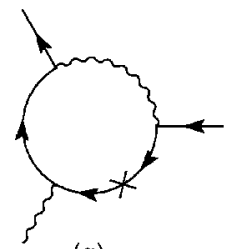

(g)

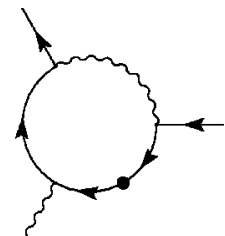

(b)

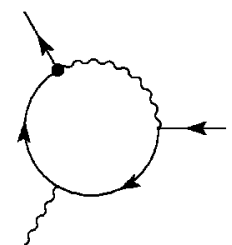

(e)

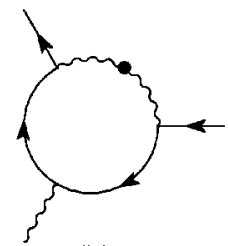

(h)

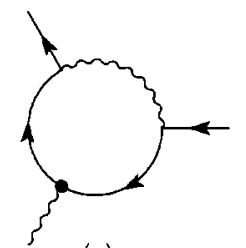

(c)

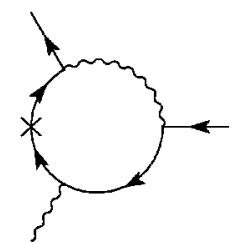

(f)

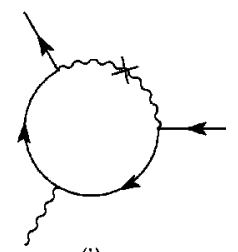

(i)
FIG. 2. Fermion-photon vertices in the QED extension. 
integrals by extending spacetime to $d=4-2 \epsilon$ dimensions in the conventional way, so that the one-loop divergent corrections to the effective Lagrangian take the form of poles in the infinitesimal parameter $\epsilon$, as usual.

Certain diagrams involve factors of $\gamma_{5}$, which introduces complications in dimensional regularization because the properties of $\gamma_{5}$ are dimension dependent. One possibility is to use the 't Hooft-Veltman definition [6] of $\gamma_{5}$, in which the $\gamma$-matrix algebra is infinite-dimensional in non-integer dimensions and the first four $\gamma^{\mu}$ are treated differently from the others. In particular, $\gamma_{5}$ anticommutes with these four while commuting with all the others:

$$
\left\{\gamma_{5}, \gamma^{\mu}\right\}=0, \quad \mu \in\{0,1,2,3\}, \quad\left[\gamma_{5}, \gamma^{\mu}\right]=0, \quad \mu \geqslant 4 .
$$

This procedure introduces a technical breaking of Lorentz invariance in all but the first four dimensions, but without introducing new physical features in our perturbation expansion because the integrals to be regularized have conventional Lorentz properties. In any case, in the present context it is simpler to adopt instead a naive $\gamma_{5}$ matrix that anticommutes with all of the other $\gamma$ matrices,

$$
\left\{\gamma_{5}, \gamma^{\mu}\right\}=0, \quad \mu \geqslant 0,
$$

which leads to errors of order $\epsilon$ in $\gamma$-matrix manipulations and hence to errors in finite terms. Since we are interested here in the divergences at one loop, all of which are simple poles in $\epsilon$, the naive $\gamma_{5}$ leaves the poles unaffected while easing calculation. Determination of the finite radiative contributions at one loop would require more care but lies beyond our present scope.

Another issue arises because the one-loop integrals span an infinite range of four-momentum. The theory (1) is known to violate stability or microcausality at sufficiently high energy and momentum, where unrenormalizable terms from Planck-scale physics become important and must be included in the analysis [3]. The Feynman rules adopted here are therefore strictly valid only over a range of energy and momentum lying below the Planck scale. We proceed in this section under the reasonable and customary assumption that any new physics entering at high scales has negligible effect on the leading-order low-energy physics described by the Lagrangian (1). A definitive result concerning the validity of this assumption would be of interest.

A technical point to note is that no external-leg propagators appear because we are calculating corrections to the oneloop effective action. External legs introduce additional complications because the full propagator at all orders in coefficients for Lorentz violation is needed to establish the asymptotic Hilbert space [2]. More attention would therefore be required to extract the finite radiative corrections to physical scattering cross sections or decay amplitudes at one loop.

We finally remark in passing that, since Lorentz symmetry is no longer respected by the theory, certain Lorentznoninvariant regularization schemes might in principle be envisaged instead. It is conceivable that a scheme chosen to respect both observer Lorentz invariance and any remaining subgroup of the particle Lorentz symmetry might offer some

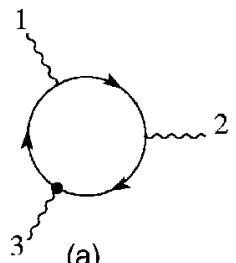

(a)

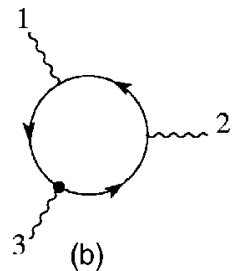

(b)
FIG. 3. Two contributions to the cubic photon interaction.

practical or conceptual advantage over more conventional regularizations. In any case, standard dimensional regularization suffices for our purposes here.

\section{B. Generalized Furry theorem and photon-interaction vertices}

Renormalizability of the QED extension at one loop requires that no divergent contributions to the three- or fourpoint photon vertices arise, since these must be absent in an Abelian gauge theory. In conventional QED, the Furry theorem [5] plays a useful role in this regard. In this section, we establish a generalized Furry theorem and use it and other calculations to prove the absence of divergent contributions to photon interactions at one loop.

In conventional QED, the Furry theorem relies on the $\gamma$-matrix structure of the photon-fermion vertex, which leads to a cancellation between two nonzero loops differing only in the direction of the charge flow. However, the QED extension (1) includes terms with more general $\gamma$-matrix structures. In this case, corresponding loops with Lorentzviolating insertions either cancel or add, depending on the charge-conjugation properties of the associated $\gamma$-matrix insertion.

As an example, consider the cubic photon vertex at one loop with an insertion of $\Gamma_{1}^{\mu}$ at one of the fermion-photon vertices in Fig. 1(c). This gives two contributions shown in Fig. 3. Take the loop momentum $k$ to be positive in the clockwise direction, and assign the $n$th external photon line a momentum $p_{n}$ and Lorentz index $\mu_{n}$. Define $k_{1}=k+p_{1}$ and $k_{12}=k+p_{1}+p_{2}$. Then, the two diagrams yield an expression proportional to

$$
\begin{gathered}
\int \frac{d^{d} k}{(2 \pi)^{d}}\left[\frac{\operatorname{Tr}\left[(\boldsymbol{k}+m) \gamma^{\mu_{1}}\left(\boldsymbol{k}_{1}+m\right) \gamma^{\mu_{2}}\left(\boldsymbol{k}_{12}+m\right) \Gamma_{1}^{\mu_{3}}\right]}{\left(k^{2}-m^{2}\right)\left(k_{1}^{2}-m^{2}\right)\left(k_{12}^{2}-m^{2}\right)}\right. \\
\left.-\frac{\operatorname{Tr}\left[\Gamma_{1}^{\mu_{3}}\left(\boldsymbol{k}_{12}-m\right) \gamma^{\mu_{2}}\left(\boldsymbol{k}_{1}-m\right) \gamma^{\mu_{1}}(\boldsymbol{k}-m)\right]}{\left(k^{2}-m^{2}\right)\left(k_{1}^{2}-m^{2}\right)\left(k_{12}^{2}-m^{2}\right)}\right] .
\end{gathered}
$$

Taking the transpose of the argument of the trace in the second term and inserting suitable factors of $C C^{-1}$, where $C$ is the charge-conjugation matrix, we can rewrite the numerator of the integrand as

$$
\operatorname{Tr}\left[(k+m) \gamma^{\mu_{1}}\left(\boldsymbol{k}_{1}+m\right) \gamma^{\mu_{2}}\left(\boldsymbol{k}_{12}+m\right)\left(\Gamma_{1}^{\mu_{3}}-\widetilde{\Gamma}_{1}^{\mu_{3}}\right)\right],
$$

where 


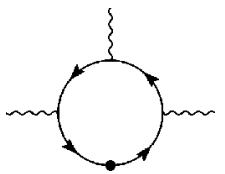

FIG. 4. Cubic photon vertex with $\Gamma_{1}^{\mu}$ propagator insertion.

$$
\begin{aligned}
\widetilde{\Gamma}_{1}^{\nu} & \equiv-\left(C \Gamma_{1}^{\nu} C^{-1}\right)^{T} \\
& =c^{\mu \nu} \gamma_{\mu}-d^{\mu \nu} \gamma_{5} \gamma_{\mu}-e^{\nu}-i f^{\nu} \gamma_{5}+\frac{1}{2} g^{\lambda \mu \nu} \sigma_{\lambda \mu}
\end{aligned}
$$

In this case, it follows that only the terms in $\Gamma_{1}^{\mu}$ associated with $C$ violation survive: those involving $I, \gamma_{5}, \gamma_{5} \gamma^{\mu}$. The usual Furry theorem is a special case for the $C$-preserving QED interaction, with $\Gamma_{1}^{\mu}$ replaced by $\gamma^{\mu}$.

If instead a factor of $\Gamma_{1}^{\mu}$ is inserted in a fermion propagator, a related argument applies. See Fig. 4. Three conventional fermion-photon vertices occur, but an extra fermion propagator appears in the loop along with another momentum factor from the insertion. Since the propagator has no net effect while the signs from the momentum insertion and the extra conventional vertex cancel, the surviving terms are the same as before.

For a four-point vertex, an extra propagator and $\gamma$ matrix appear relative to the three-point vertex. These combine to give an overall relative sign. It therefore follows that coefficients surviving in a three-point function are eliminated in the corresponding four-point function, and vice versa. These arguments can be generalized to include insertions of $M_{1}$ in fermion propagators and arbitrary numbers of photon legs around the loop.

The generalization of the Furry theorem thus shows that there are no contributions proportional to $b_{\mu}, c_{\mu \nu}$, or $g_{\lambda \mu \nu}$ for odd numbers of photon legs on a fermion loop, while there are no contributions proportional to $a_{\mu}, d_{\mu \nu}, e_{\mu}, f_{\mu}$, or $H_{\mu \nu}$ for even numbers of photon legs. The contributions from other pairs of diagrams with opposing fermion loops must be explicitly calculated and typically are nonzero. This applies to both finite and divergent corrections. For example, it is no longer necessarily the case that one-loop radiative corrections vanish for $n$-point photon $S$-matrix amplitudes with odd $n$. Even for the 3-point photon vertex, there could now be a nonzero amplitude. Although it lies outside our present scope, it would be interesting to evaluate these radiative effects and consider possible phenomenological implications.

To investigate renormalizability, it is necessary to calculate explicitly the divergent one-loop contributions to the three- and four-point photon vertices for those cases where the generalized Furry theorem allows a nonzero answer. For the cubic vertex there are three vertices and three propagators, so any nonzero divergent contribution would occur three times. The resulting permutations are illustrated in Fig. 5 for the case of propagator insertions. Any diagram with an $H_{\mu \nu}$ insertion is finite either because of the dimensionality of $H_{\mu \nu}$ or because the trace of an odd number of $\gamma$-matrices vanishes. Explicit calculation reveals that no divergences

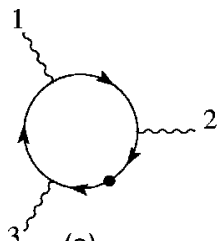

(a)

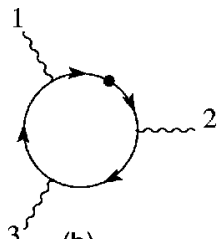

(b)

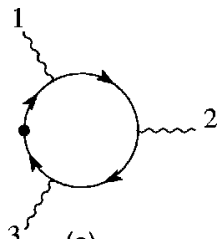

(c)
FIG. 5. Permutations for the cubic photon vertex.

proportional to the other coefficients for Lorentz violation occur either.

For the quartic photon vertex, the generalized Furry theorem and the dimensionality of $b_{\mu}$ together imply that the only potential divergences arise from insertions of $c_{\mu \nu}$ or $g_{\lambda \mu \nu}$. In this case, there are four permutations of each type of insertion. The contributions involving $g_{\lambda \mu \nu}$ are zero because the trace of an odd number of $\gamma$ matrices vanishes. Once again, explicit calculation reveals that no divergence proportional to $c_{\mu \nu}$ occurs either, as required for renormalizability.

\section{Propagator corrections}

In this subsection, we provide the results of our calculations for one-loop corrections to the photon and fermion propagators. The calculational methods parallel the conventional case, so for brevity we restrict the discussion largely to the presentation of results.

For the photon propagator, the complete one-loop divergence including the standard QED result is

$$
\begin{aligned}
\bar{\omega}_{\mu \nu}(p)= & \frac{4 q^{2}}{3} I_{0}\left[\left(p_{\mu} p_{\nu}-p^{2} \eta_{\mu \nu}\right)-\left(c_{\mu \nu}+c_{\nu \mu}\right) p^{2}\right. \\
& -2 c_{\alpha \beta} p^{\alpha} p^{\beta} \eta_{\mu \nu}+\left(c_{\mu \alpha}+c_{\alpha \mu}\right) p^{\alpha} p_{\nu} \\
& \left.+\left(c_{\nu \alpha}+c_{\alpha \nu}\right) p^{\alpha} p_{\mu}\right],
\end{aligned}
$$

where $I_{0}=i / 16 \pi^{2} \epsilon$. Only the symmetric part of $c_{\mu \nu}$ contributes. Corrections coming from diagrams involving vertex and propagator insertions of $g^{\lambda \mu \nu}$ and the antisymmetric part of $c^{\mu \nu}$ cancel. All other potential divergent corrections to this propagator can be shown to vanish, using arguments similar to those in Sec. III B. Note that $\bar{\omega}_{\mu \nu}$ is symmetric and gauge invariant, $p^{\mu} \bar{\omega}_{\mu \nu}=0$. The result (10) agrees with the original calculation in Ref. [2].

For the fermion propagator, the usual QED correction is

$$
\Sigma_{\mathrm{QED}}(p)=q^{2} I_{0}\left[(1+\xi) \gamma_{\mu} p^{\mu}-(4+\xi) m\right]
$$

The divergent corrections $\Sigma_{x}$ arising from all possible insertions of an $x$ term in the standard one-loop QED diagram are given by

$$
\begin{aligned}
\Sigma_{M_{1}}(p) & =-q^{2} I_{0}\left[(1+\xi)\left(a_{\mu}+\gamma_{5} b_{\mu}\right) \gamma^{\mu}+\frac{\xi}{2} H_{\mu \nu} \sigma^{\mu \nu}\right], \\
\Sigma_{c}(p) & =\frac{q^{2}}{3} I_{0}\left[(3 \xi-1) c_{\nu \mu} p^{\mu} \gamma^{\nu}-4 c_{\mu \nu} p^{\mu} \gamma^{\nu}\right],
\end{aligned}
$$




$$
\begin{aligned}
\Sigma_{d}(p)= & \frac{q^{2}}{3} I_{0}\left[(3 \xi-1) d_{\nu \mu} p^{\mu} \gamma_{5} \gamma^{\nu}-4 d_{\mu \nu} p^{\mu} \gamma_{5} \gamma^{\nu}\right. \\
& \left.-3 m \epsilon^{\alpha \beta \mu \nu} d_{\alpha \beta} \sigma_{\mu \nu}\right], \\
\Sigma_{e}(p)= & q^{2} I_{0}\left[(1+\xi) e_{\mu} p^{\mu}-3 m e_{\mu} \gamma^{\mu}\right], \\
\Sigma_{f}(p)= & q^{2} I_{0}(1+\xi) i f_{\mu} p^{\mu} \gamma_{5}, \\
\Sigma_{g}(p)= & \frac{q^{2}}{2} I_{0}\left[(\xi-1) g_{\lambda \mu \nu} p^{\nu} \sigma^{\lambda \mu}-2 g_{\lambda \mu \nu} p^{\mu} \sigma^{\lambda \nu}\right. \\
& \left.+2 g_{\mu \alpha}{ }^{\alpha} p_{\nu} \sigma^{\mu \nu}-m g^{\alpha \beta \gamma} \epsilon_{\alpha \beta \gamma \mu} \gamma_{5} \gamma^{\mu}\right], \\
\Sigma_{k_{A F}}(p)= & 3 q^{2} I_{0}\left(k_{A F}\right)_{\nu} \gamma_{5} \gamma^{\nu}, \\
\Sigma_{k_{F}}(p)= & \frac{4 q^{2}}{3} I_{0}\left(k_{F}\right)_{\mu \alpha \nu}{ }^{\alpha} p^{\mu} \gamma^{\nu} .
\end{aligned}
$$

\section{Quadratic-term renormalization factors}

To renormalize the quadratic terms, we must redefine the bare fields and the fermion mass in terms of renormalized ones,

$$
\psi_{B}=\sqrt{Z_{\psi}} \psi, \quad A_{B}^{\mu}=\sqrt{Z_{A}} A^{\mu}, \quad m_{B}=Z_{m} m,
$$

and the bare coefficients for Lorentz violation in terms of renormalized ones,

$$
\begin{array}{r}
a_{B \mu}=\left(Z_{a}\right)_{\mu}{ }^{\alpha} a_{\alpha}, \quad b_{B \mu}=\left(Z_{b}\right)_{\mu}{ }^{\alpha} b_{\alpha}, \\
c_{B \mu \nu}=\left(Z_{c}\right)_{\mu \nu}{ }^{\alpha \beta} c_{\alpha \beta}, \quad d_{B \mu \nu}=\left(Z_{d}\right)_{\mu \nu}{ }^{\alpha \beta} d_{\alpha \beta}, \\
e_{B \mu}=\left(Z_{e}\right)_{\mu}{ }^{\alpha} e_{\alpha}, \quad f_{B \mu}=\left(Z_{f}\right)_{\mu}{ }^{\alpha} f_{\alpha}, \\
g_{B \lambda \mu \nu}=\left(Z_{g}\right)_{\lambda \mu \nu}{ }^{\alpha \beta \gamma} g_{\alpha \beta \gamma}, \quad H_{B \mu \nu}=\left(Z_{H}\right)_{\mu \nu}{ }^{\alpha \beta} H_{\alpha \beta}, \\
\left(k_{A F}\right)_{B \mu}=\left(Z_{k_{A F}}\right)_{\mu}{ }^{\alpha}\left(k_{A F}\right)_{\alpha}, \\
\left(k_{F}\right)_{B \kappa \lambda \mu \nu}=\left(Z_{k_{F}}\right)_{\kappa \lambda \mu \nu}{ }^{\alpha \beta \gamma \delta}\left(k_{F}\right)_{\alpha \beta \gamma \delta} .
\end{array}
$$

In this section, a subscript $B$ is added to bare quantities where needed to distinguish them from renormalized ones.

An analysis of Eqs. (11) and (12) leads to the following expressions for the above renormalization factors:

$$
\begin{aligned}
Z_{\psi} & =1-\frac{q^{2}}{16 \pi^{2} \epsilon}(1+\xi), \\
Z_{A} & =1-\frac{q^{2}}{12 \pi^{2} \epsilon}, \\
Z_{m} & =1-\frac{3 q^{2}}{16 \pi^{2} \epsilon}, \\
\left(Z_{a}\right)_{\mu}{ }^{\alpha} a_{\alpha} & =a_{\mu}-\frac{3 q^{2}}{16 \pi^{2} \epsilon} m e_{\mu},
\end{aligned}
$$


relevant corrections come from $\left.\Sigma_{e}(p)\right|_{m=0}$. Including the effects arising from the wave-function renormalization $Z_{\psi}$ reveals that no renormalization of $e^{\mu}$ is needed at one loop. A more involved example is given by the corrections to $c_{\mu \nu}$, which arise from both $\Sigma_{c}(p)$ and $\Sigma_{k_{F}}(p)$. Incorporating also the wave-function renormalization $Z_{\psi}$ leads to the above expression for $\left(Z_{c}\right)_{\mu \nu}{ }^{\alpha \beta} c_{\alpha \beta}$. As a final example, consider $\left(Z_{k_{F}}\right)_{\kappa \lambda \mu \nu}{ }^{\alpha \beta \gamma \delta}$. Here, it is useful to note that the term $-2 p^{\alpha} p^{\beta} c_{\alpha \beta} \eta_{\mu \nu}$ in Eq. (10) must cancel a correction to the tree-level $\left(k_{F}\right)_{\mu \nu \alpha \beta}$ term, and hence the correction itself must also have the symmetry of the Riemann tensor. Implementing this requirement reveals that all the divergent terms cancel simultaneously provided $Z_{A}$ takes the same form as in conventional QED at one loop.

\section{E. Vertex corrections and Ward identities}

The remaining 1PI diagrams arise in connection with the one-loop three-point vertex. This section presents the results of our calculations of the associated diagrams.

For this vertex, the standard QED divergence at one loop is recovered:

$$
\Gamma_{\mathrm{QED}}^{\mu}=-q^{3} I_{0}(1+\xi) \gamma^{\mu} .
$$

The divergent corrections $\Gamma_{x}^{\mu}$ arising from all possible single insertions of an $x$ term in the standard one-loop QED threepoint vertex are found to be

$$
\begin{aligned}
\Gamma_{M_{1}}^{\mu}= & 0, \\
\Gamma_{c}^{\mu}= & -\frac{q^{3}}{3} I_{0}\left[(3 \xi-1) c^{\alpha \mu} \gamma_{\alpha}-4 c^{\mu \alpha} \gamma_{\alpha}\right], \\
\Gamma_{d}^{\mu}= & -\frac{q^{3}}{3} I_{0}\left[(3 \xi-1) d^{\alpha \mu} \gamma_{5} \gamma_{\alpha}-4 d^{\mu \alpha} \gamma_{5} \gamma_{\alpha}\right], \\
\Gamma_{e}^{\mu}= & -q^{3} I_{0}(1+\xi) e^{\mu}, \\
\Gamma_{f}^{\mu}= & -q^{3} I_{0}(1+\xi) i f^{\mu} \gamma_{5}, \\
\Gamma_{g}^{\mu}= & -\frac{q^{3}}{2} I_{0}\left[(\xi-1) g^{\alpha \beta \mu} \sigma_{\alpha \beta}+2 g^{\alpha}{ }_{\beta}{ }^{\beta} \sigma_{\alpha \mu}\right. \\
& \left.+2 g^{\alpha \mu \beta} \sigma_{\beta \alpha}\right], \\
\Gamma_{k_{A F}}^{\mu}= & 0, \\
\Gamma_{k_{F}}^{\mu}= & -\frac{4 q^{3}}{3} I_{0}\left(k_{F}\right)_{\mu \alpha \beta}{ }^{\alpha} \gamma^{\beta} .
\end{aligned}
$$

The reader is cautioned not to confuse the standard notation $\Gamma_{x}^{\mu}$ for the divergent 3-vertex contributions with the quantities $\Gamma^{\mu}$ and $\Gamma_{1}^{\mu}$ in Eqs. (1) and (2).

Taking into account the results (15), renormalization of the QED vertex correction (17) yields

$$
q_{B}=Z_{q} q, \quad Z_{q}=1+\frac{q^{2}}{24 \pi^{2} \epsilon} .
$$

This is in accordance with the usual QED Ward identity,

$$
Z_{q} \sqrt{Z_{A}}=1 \text {. }
$$

Note that the $Z$ factors for the couplings are all independent of the gauge parameter $\alpha=(1-\xi)$, as expected.

At this stage no more parameters can be renormalized, so all the remaining divergent corrections $\Gamma_{x}^{\mu}$ must be made finite by the $Z$ factors already defined. Inspection shows that this is indeed the case, provided the Ward identity holds. We can therefore conclude that the theory is multiplicatively renormalizable and that it remains gauge invariant at one loop.

\section{RENORMALIZATION GROUP AND BETA FUNCTIONS}

Given the results for the one-loop divergences presented in Sec. III, several interesting issues become amenable to analysis. In particular, we can initiate a study of the behavior of the Lorentz- and $C P T$-violating QED extension over a large range of energy scales. This offers, for example, partial insight about the absolute and relative sizes of the coefficients for Lorentz violation and hence is of value from both theoretical and experimental perspectives.

In this section, we begin with some preliminary remarks delineating the framework for our analysis. We then obtain the beta functions and solve the renormalization-group equations for the running of the coefficients for Lorentz violation. Some implications of these results are discussed.

\section{A. Framework}

Any regularization scheme naturally introduces a mass scale $\mu$. In the Pauli-Villars scheme it is the momentum cutoff, while in dimensional regularization it is the unit of mass required to maintain a dimensionless action in $d$ dimensions. In a conventional field theory, the bare Green functions are independent of the value chosen for $\mu$. However, they are related by multiplicative renormalizability to the renormalized Green functions, which may depend on $\mu$ as a consequence of the regularization procedure.

In perturbation theory, the coefficients of a renormalized Green function typically depend on logarithms $\ln \left(p^{2} / \mu^{2}\right)$ of the momentum $p$. As a result, if $\sqrt{p^{2}}$ is very different from $\mu$, perturbation theory can become invalid even for small couplings. To study the physics at momenta much larger than $\mu$, the Green function must be expressed in terms of a new renormalization mass $\mu^{\prime} \approx \sqrt{p^{2}}$ chosen to keep the logarithms small, so that it is justifiable to neglect higher-order terms.

In the present case, we are interested in the behavior of the coefficients for Lorentz violation over a large range of scales. We are therefore interested in the dependence of the renormalized Green functions on the renormalization mass scale $\mu$. A standard procedure to obtain this is to solve the renormalization-group equation at the appropriate order in 
perturbation theory [20,21]. The solution can be expressed through the dependence on $\mu$ of the renormalized running couplings $x(\mu)$, where $x$ generically denotes parameters in the theory.

Under the assumption that the Lorentz- and $C P T$-violating QED extension is a multiplicatively renormalizable theory to all orders, the usual derivation of the renormalization-group equation can be followed. For an $n$-point Green function $\Gamma(n)$, we can write

$$
\Gamma_{B}(n)\left[p, x_{B}\right]=Z^{-n / 2}[x(\mu), \mu] \Gamma(n)[p, x(\mu), \mu],
$$

where $x$ includes the coefficients for Lorentz violation as well as $m$ and $q$. The factor of $Z^{-n / 2}$ arises from wavefunction renormalization of the external legs of the Green function. Noting that the left-hand side of this equation is independent of $\mu$, differentiation with respect to $\mu$ yields

$$
0=\mu \frac{d}{d \mu}\left\{Z^{-n / 2}[x(\mu), \mu] \Gamma(n)[p, x(\mu), \mu]\right\} .
$$

This is the renormalization-group equation, which provides a nontrivial constraint on $\Gamma(n)$ through the explicit $\mu$ dependence.

For a one-loop calculation, it suffices to impose Eq. (22) only to one-loop order. If the running couplings become large enough, the perturbative approach fails. However, in the region where the couplings remain small, the accuracy of the perturbative approximation is improved compared to using fixed couplings. This improvement can be attributed to a partial resummation of the perturbation series that includes leading logarithmic corrections to all orders in perturbation theory.

Despite the link between higher loops and renormalization-group improvement, the explicit one-loop solution of Eq. (22) only uses multiplicative renormalizability at one loop. Since one-loop multiplicative renormalizability of the Lorentz- and $C P T$-violating QED extension is proved in the previous section, it follows that we can perform one-loop renormalization-group calculations without meeting practical obstacles. In effect, this procedure makes the reasonable assumption that the couplings remain small and hence that the perturbation approximation is valid. However, the derivation of the renormalization-group equation outlined above shows that adopting this calculational procedure also tacitly assumes that the QED extension is multiplicatively renormalizable at all orders. Resolving this multiloop issue lies well beyond our present scope, so we restrict ourselves here to a few pertinent remarks.

It is known that operators of mass dimension greater than four are required in the full QED extension for causality and stability at Planck-related energy scales [3]. Although such operators are unnecessary at low energy, where the theory (1) holds sway, their presence implies that the full theory is unrenormalizable in the usual sense. However, this may be irrelevant in the underlying Planck-scale theory. For example, difficulties with ultraviolet properties are absent in some string theories even though the corresponding particle field theories appear to have unrenormalizable terms at leading order in the string tension. In the present context, the existence of these operators suggests that the Lagrangian (1) can be regarded as an effective low-energy theory. From this perspective, the key assumption made in applying Eq. (22) at one loop is that the theory remains valid as an effective field theory at this order. Then, any problems arising from unrenormalizability at higher loops would be suppressed at low energies, allowing one-loop calculations to be performed as though the theory were fully multiplicatively renormalizable. Unrenormalizable effects entering at some multiloop level might cause loss of predictability at that order in perturbation theory, but lower-order predictions would remain valid in the region where the corresponding running couplings are small.

For definiteness, we proceed in what follows under the reasonable and practical assumption that it is meaningful to apply Eq. (22) at one loop. Although beyond our present scope, it would be interesting either to prove all-orders multiplicative renormalizability of the QED extension or to determine the formal regime of validity of the results to be presented below.

\section{B. The beta functions}

Given a theory with a set $\left\{x_{j}\right\}, j=1,2, \ldots, N$, of running parameters, the beta function [21] for a specific parameter $x_{j}$ is

$$
\beta_{x_{j}} \equiv \mu \frac{d x_{j}}{d \mu},
$$

where $\mu$ is the renormalization mass parameter relevant to the regularization method. In dimensional regularization with minimal subtraction, the beta function for a given parameter can be calculated directly from the simple $\epsilon$ pole in the corresponding $Z$ factor $[22,23]$. In this subsection, we summarize the procedure and use it to obtain all the one-loop beta functions for the Lorentz- and $C P T$-violating QED extension.

For each parameter $x_{j}$, define the $Z$ factor $Z_{x_{j}}$ by

$$
x_{j B}=\mu^{\rho_{x_{j}} \epsilon} Z_{x_{j}} x_{j},
$$

where the factor $\mu^{\rho_{x_{j}}}$ ensures that the renormalized parameter $x_{j}$ has the same mass dimension as its corresponding bare parameter. In $d=4-2 \epsilon$ dimensions, the fields $\psi$ and $A^{\mu}$ have mass dimension $(3-2 \epsilon) / 2$ and $(1-\epsilon)$, respectively. We therefore find

$$
\rho_{q}=1, \quad \rho_{m}=\rho_{\Gamma_{1}}=\rho_{M_{1}}=0 .
$$

In minimal subtraction, only the divergent terms in the regulated integrals are subtracted. Since these divergent terms are poles in $\epsilon$, any given $Z$ factor takes the generic form

$$
Z_{x_{j}} x_{j}=x_{j}+\sum_{n=1}^{\infty} \frac{a_{n}^{j}}{\epsilon^{n}}
$$

It can then be shown that [22] 


$$
\beta_{x_{j}}=\lim _{\epsilon \rightarrow 0}\left[-\rho_{x_{j}} a_{1}^{j}+\sum_{k=1}^{N} \rho_{x_{k}} x_{k} \frac{\partial a_{1}^{j}}{\partial x_{k}}\right]
$$

which involves only the simple pole $a_{1}^{j}$.

Among the $\rho_{x_{j}}$, only $\rho_{q}$ is nonzero in the present case. Equation (27) therefore implies that each beta function other than $\beta_{q}$ is nontrivial only due to the $q$ dependence of the corresponding $Z$ factor. The running of all the coefficients for Lorentz violation at any loop order is therefore driven by the standard QED running of the charge $q$. This result is expected because the interactions in the theory (1) all arise from the minimal coupling in the covariant derivative and are therefore controlled only by the charge $q$, even though the perturbation theory is also an expansion in the coefficients for Lorentz violation.

Using the expression (27), we obtain the following set of results for all the beta functions in the Lorentz- and $C P T$-violating QED extension:

$$
\begin{aligned}
\beta_{m}= & -\frac{3 q^{2}}{8 \pi^{2}} m, \quad \beta_{q}=\frac{q^{3}}{12 \pi^{2}}, \\
\left(\beta_{a}\right)_{\mu}= & -\frac{3 q^{2}}{8 \pi^{2}} m e_{\mu}, \\
\left(\beta_{b}\right)_{\mu}= & -\frac{q^{2}}{16 \pi^{2}}\left[m g^{\alpha \beta \gamma} \epsilon_{\alpha \beta \gamma \mu}-6\left(k_{A F}\right)_{\mu}\right], \\
\left(\beta_{c}\right)_{\mu \nu}= & \frac{q^{2}}{6 \pi^{2}}\left[c_{\mu \nu}+c_{\nu \mu}-\left(k_{F}\right)_{\mu \alpha \nu}{ }^{\alpha}\right], \\
\left(\beta_{d}\right)_{\mu \nu}= & \frac{q^{2}}{6 \pi^{2}}\left(d_{\mu \nu}+d_{\nu \mu}\right), \quad\left(\beta_{e}\right)_{\mu}=\left(\beta_{f}\right)_{\mu}=0, \\
\left(\beta_{g}\right)_{\lambda \mu \nu}= & \frac{q^{2}}{8 \pi^{2}}\left(2 g_{\lambda \mu \nu}-g_{\nu \lambda \mu}+g_{\nu \mu \lambda}-\eta_{\mu \nu} g_{\lambda \alpha}{ }^{\alpha}\right. \\
& \left.+\eta_{\lambda \nu} g_{\mu \alpha}{ }^{\alpha}\right), \\
& \left.-\frac{1}{2} \eta_{\nu \lambda}\left(c_{\mu \kappa}+c_{\kappa \mu}\right)\right] . \\
\left(\beta_{H}\right)_{\mu \nu}= & \frac{q^{2}}{8 \pi^{2}}\left(H_{\mu \nu}-2 m d^{\alpha \beta} \epsilon_{\alpha \beta \mu \nu}\right), \\
\left(\beta_{k_{A F}}\right)_{\mu}= & \frac{q^{2}}{6 \pi^{2}}\left(k_{A F}\right)_{\mu}, \\
\kappa \lambda \mu \nu & \frac{q^{2}}{6 \pi^{2}}\left[k_{F \kappa \lambda \mu \nu}-\frac{1}{2} \eta_{\mu \kappa}\left(c_{\nu \lambda}+c_{\lambda \nu}\right)\right. \\
& \left.+c_{\mu \lambda}+c_{\lambda \mu}\right)+\frac{1}{2} \eta_{\mu \lambda}\left(c_{\nu \kappa}+c_{\kappa \nu}\right) \\
& \\
&
\end{aligned}
$$

Through the definition (23), these beta functions specify a complete set of coupled partial-differential equations govern- ing the one-loop running of the coefficients for Lorentz violation and other parameters in the theory.

\section{Running couplings}

To solve the coupled partial-differential equations (28) for the running parameters $x_{j}(\mu)$, boundary conditions are required. We provide these as the values

$$
x_{j 0} \equiv x_{j}\left(\mu_{0}\right)
$$

of the parameters $x_{j}$ at the scale $\mu_{0}$. In what follows, it is convenient to define the quantity

$$
Q(\mu) \equiv 1-\frac{q_{0}^{2}}{6 \pi^{2}} \ln \frac{\mu}{\mu_{0}},
$$

which controls the running of the usual QED charge with the scale $\mu$.

The first two of Eqs. (28) yield

$$
q(\mu)^{2}=Q^{-1} q_{0}^{2}, \quad m(\mu)=Q^{9 / 4} m_{0},
$$

which is the standard QED result. Substituting these expressions in the remaining equations permits a complete integration. As an example, consider the coefficient $d_{\mu \nu}$. Constructing the beta function for $d_{\mu \nu}+d_{\nu \mu}$ gives

$$
\frac{d}{d \ln \mu}\left(d+d^{T}\right)=\frac{q_{0}^{2}}{3 \pi^{2}} Q^{-1}\left(d+d^{T}\right)
$$

which can be rewritten as

$$
\frac{d}{d \ln \mu}\left[Q^{2}\left(d+d^{T}\right)\right]=0 .
$$

Therefore, the symmetric part of $d_{\mu \nu}$ runs like $Q^{-2}$. Similarly, we find the antisymmetric part has no running. Combining the two gives the running of $d_{\mu \nu}$.

In this way, we find that the coefficients for Lorentz violation run as follows:

$$
\begin{aligned}
a_{\mu}= & a_{0 \mu}-m_{0}\left(1-Q^{9 / 4}\right) e_{0 \mu}, \\
b_{\mu}= & b_{0 \mu}-\frac{1}{6} m_{0}\left(1-Q^{9 / 4}\right) g_{0}^{\alpha \beta \gamma} \epsilon_{\alpha \beta \gamma \mu} \\
& -\frac{9}{4} \ln Q\left(k_{A F}\right)_{0 \mu}, \\
c_{\mu \nu}= & c_{0 \mu \nu}-\frac{1}{3}\left(1-Q^{-3}\right)\left[c_{0 \mu \nu}+c_{0 \nu \mu}-\left(k_{F}\right)_{0 \mu \alpha \nu}{ }^{\alpha}\right], \\
d_{\mu \nu}= & d_{0 \mu \nu}-\frac{1}{2}\left(1-Q^{-2}\right)\left(d_{0 \mu \nu}+d_{0 \nu \mu}\right), \\
e_{\mu}= & e_{0 \mu}, \quad f_{\mu}=f_{0 \mu},
\end{aligned}
$$




$$
\begin{aligned}
g_{\lambda \mu \nu}= & g_{0 \lambda \mu \nu}-\frac{1}{3}\left(1-Q^{-9 / 4}\right)\left(2 g_{0 \lambda \mu \nu}-g_{0 \nu \lambda \mu}+g_{0 \nu \mu \lambda}\right. \\
& \left.-\eta_{\mu \nu} g_{0 \lambda \alpha}{ }^{\alpha}+\eta_{\nu \lambda} g_{0 \mu \alpha}{ }^{\alpha}\right) \\
H_{\mu \nu}= & H_{0 \mu \nu}+\frac{1}{2} m_{0}\left(1-Q^{9 / 4}\right) d_{0}^{\alpha \beta} \epsilon_{\alpha \beta \mu \nu} \\
\left(k_{A F}\right)_{\mu}= & Q^{-1}\left(k_{A F}\right)_{0 \mu} \\
\left(k_{F}\right)_{\kappa \lambda \mu \nu}= & \left(k_{F}\right)_{0 \kappa \lambda \mu \nu}+\frac{1}{6}\left(1-Q^{-3}\right) \\
& \times\left\{\eta_{\mu \kappa}\left[c_{\nu \lambda}+c_{\lambda \nu}-\left(k_{F}\right)_{0 \nu \alpha \lambda}{ }^{\alpha}\right]\right. \\
& -\eta_{\nu \kappa}\left[c_{\mu \lambda}+c_{\lambda \mu}+\left(k_{F}\right)_{0 \mu \alpha \lambda}^{\alpha}\right] \\
& -\eta_{\mu \lambda}\left[c_{\nu \kappa}+c_{\kappa \nu}+\left(k_{F}\right)_{0 \nu \alpha \kappa}^{\alpha}\right] \\
& \left.+\eta_{\nu \lambda}\left[c_{\mu \kappa}+c_{\kappa \mu}-\left(k_{F}\right)_{0 \mu \alpha \kappa}{ }^{\alpha}\right]\right\}
\end{aligned}
$$

Some coefficients increase or decrease with $Q$ while some are unaffected, including irreducible combinations such as the totally antisymmetric part of $g_{\lambda \mu \nu}$. Note that the mixings of coefficients for Lorentz violation displayed in these results are consistent with the symmetry-based predictions given at the end of Sec. II.

\section{Some implications}

The expressions (34) display a range of behavior for the running of the coefficients for Lorentz violation. All the running is controlled by the single function $Q(\mu)$ given in Eq. (30), but the powers of $Q(\mu)$ involved range from -3 to 9/4. In this section, we comment on some implications of this behavior.

Note first that the calculations above are performed for the Lorentz- and CPT-violating QED extension with a single Dirac fermion. However, the full standard-model extension involves additional interactions, three generations of chiral fermion multiplets, a scalar field, and several intermeshed sets of coefficients for Lorentz violation. All these would affect the structure of the solutions (34). A definitive understanding of the physical implications of the running of the coefficients for Lorentz violation must therefore await a complete analysis within the standard-model extension. Nonetheless, despite these caveats, some meaningful physical insight can be obtained.

We focus here on the behavior of $Q(\mu)$ from low energies to the Planck scale. A key factor in determining this behavior is the value of the fermion charge $q_{0}$ at the reference scale $\mu_{0}$, which controls the size of the coefficient of the logarithm in Eq. (30). In a realistic theory, the running of the QED charge $q$ involves all charged fermions, so the factor $q_{0}^{2}$ in Eq. (30) must be replaced with a quantity involving a sum over squared charges of all fermions participating in the loops. At sufficiently high energies, this includes all known fermions in the standard model and possibly others predicted by the theory but as yet unobserved. Any charged scalars in the theory could also play a role. In many theories, it may

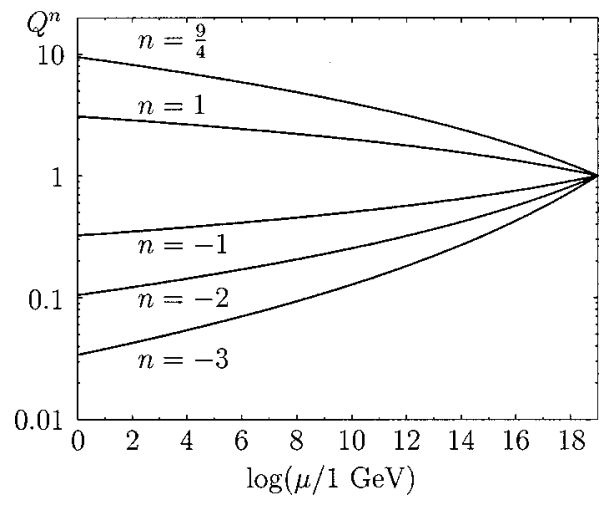

FIG. 6. Variation of the function $Q(\mu)^{n}$.

also be necessary to account for the possible embedding of the charge $\mathrm{U}(1)$ group in a larger unification gauge group.

Since the function $Q(\mu)$ is sensitive to the coefficient of the logarithm, the combination of the above factors implies that different physically realistic theories can produce a variety of behaviors for $Q(\mu)$. For the standard-model fermions alone, the sum of the squared charges is about 0.7 , while the Landau pole for running between the electroweak scale $m_{e w} \simeq 250 \mathrm{GeV}$ and the Planck scale $M_{P} \simeq 2 \times 10^{19} \mathrm{GeV}$ occurs at a corresponding factor of about 1.5. For simplicity and to gain basic insight, we consider explicitly the case where this factor is chosen to be 1 .

Figure 6 shows the various powers of the function $Q(\mu)$ for this case, $q_{0}^{2}=1$, plotted as a logarithmic function of $\mu$ from $m_{e w}$ to $M_{P}$. In a simple scenario for Lorentz and $C P T$ violation, such as might arise in spontaneous symmetry breaking [9], it is conceivable that near the Planck scale all nonzero coefficients for Lorentz violation are the same order of magnitude in Planck units. Then, Fig. 6 can be directly interpreted in terms of the divergence of renormalizationgroup trajectories at low energies. If instead the coefficients for Lorentz violation start at different sizes at the Planck scale, then matching the curves in Fig. 6 to the trajectories of the running couplings requires shifts. In any case, the figure shows that some coefficients for Lorentz violation increase while others decrease as the energy scale changes, with possible relative changes of several orders of magnitude.

The rate of running of the coefficients for Lorentz violation is relatively small, as is to be expected from the logarithmic scale dependence and from the slow running of the QED coupling $q$. This running would therefore be insufficient by itself to account for the heavy suppression of coefficients for Lorentz violation necessary for compatibility with existing experimental bounds. If not already present at the Planck scale, any suppression must be driven by some other factor relevant between the electroweak and the Planck scales. In fact, it is known that unrenormalizable terms become important for consistency as the Planck scale is approached [3]. We conjecture that, in mixing with other coefficients in the renormalization-group equations, these terms could suffice to provide the necessary suppression at the electroweak scale. A rapid running of coefficients for Lorentz violation with negative mass dimension is consistent with expectations from the results (34), which indicate that di- 
mensionless coefficients increase towards the Planck scale while the massive ones decrease or remain unchanged. Although it lies outside our present scope, it would be interesting to investigate further this line of reasoning in the context of explicit models.

In the standard-model extension, the coefficients for Lorentz violation may vary with the particle species. Figure 6 shows that the running of $Q(\mu)$ with the scale $\mu$ can suffice to induce a significant range of values for the various coefficients specific to a given species, even if all these coefficients are comparable at some large scale. The figure also suggests that conventional running alone cannot separate coefficients for a given species by more than several orders of magnitude. In contrast, for different species of the same nonzero charge, no separation is induced between coefficients for Lorentz violation of a specific type.

Note also that the running of the coefficients in the full standard-model extension must also be controlled by factors $Q_{2}(\mu)$ and $Q_{3}(\mu)$ associated with the $\mathrm{SU}(2)$ and $\mathrm{SU}(3)$ gauge groups, respectively. In some theories, the SU(3) coupling runs faster than the others, which suggests a larger spread in the quark-sector coefficients for Lorentz violation and emphasizes the value of a range of tests sensitive to different coefficients for hadrons [11-15]. In other theories, the $\mathrm{U}(1)$ coupling runs the fastest, so the greatest spread may be in the charged-lepton sector, emphasizing the need for different measurements with electrons $[16,17]$ and muons [19]. Taken together, all these considerations underline the importance of performing experiments measuring a wide variety of different coefficients, both within a given species and across different sectors of the standard-model extension.

\section{SUMMARY}

In this paper we have shown the one-loop multiplicative renormalizability of the general Lorentz- and $C P T$-violating QED extension (1). A generalized Furry theorem has been obtained and used to prove the absence of one-loop divergences in the cubic and quartic photon vertices. The divergent one-loop corrections to the photon propagator are given in Eq. (10), while those to the fermion propagator are in Eqs. (11) and (12). The associated renormalization factors are in Eqs. (15) and (19). The divergent one-loop corrections to the fermion-photon vertex are given in Eqs. (17) and (18). The usual Ward identities are found to hold at this order.

We have also initiated an investigation into the running of the coefficients for Lorentz violation and the renormalization-group equations. Following some discussion of the framework, the one-loop beta functions are obtained as Eq. (28). The associated partial-differential equations are solved, and the running couplings are provided in Eqs. (30), (31), and (34). We show that these equations imply that different coefficients for Lorentz violation typically run differently between the electroweak and Planck scales and can lead to a spread of several orders of magnitude over this range. Our work emphasizes the value of developing tests to measure many different coefficients for Lorentz and $C P T$ violation, both for specific field species and across all sectors of the standard-model extension.

\section{ACKNOWLEDGMENTS}

This work was supported in part by the United States Department of Energy under grant No. DE-FG0291ER40661. A.P. wishes to thank John Gracey and D.R.T. Jones for useful discussions.

\section{APPENDIX: FEYNMAN RULES}

This appendix provides the Feynman rules appropriate for our one-loop calculations using the theory (1) with the standard gauge-fixing term $-(\partial \cdot A)^{2} / 2 \alpha$. They are linear in the coefficients for Lorentz and $C P T$ violation.

The fermion propagator is

$$
\longrightarrow=i \frac{\left(\gamma_{\mu} p^{\mu}+m\right)}{p^{2}-m^{2}},
$$

where the momentum $p^{\mu}$ is understood to travel in the direction of the charge arrow shown in the diagram. The coefficients for Lorentz and $C P T$ violation contained in $\Gamma_{1}^{\mu}$ and $M_{1}$ lead to insertions in the fermion propagator:

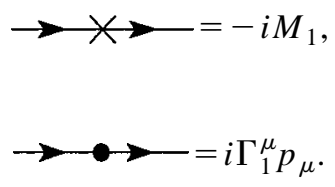

For a photon of momentum $p^{\mu}$, the propagator is

$$
\mu \sim \sim \frac{i}{p^{2}}\left(\eta_{\mu \nu}+\frac{p_{\mu} p_{v}}{p^{2}} \xi\right)
$$

where $\xi=\alpha-1$. The coefficients for Lorentz and $C P T$ violation yield two types of insertion on this propagator:

$$
\mu \sim \nu=-2 i p^{\alpha} p^{\beta}\left(k_{F}\right)_{\alpha \mu \beta \nu},
$$

The momentum $p^{\mu}$ for the $\left(k_{A F}\right)^{\alpha}$ insertion is taken to travel from the $\mu$ index to the $\nu$ index. Note that the Feynman diagram is symmetric: the antisymmetry of $\epsilon_{\alpha \mu \beta \nu}$ under $\mu, \nu$ interchange is compensated by the reversal of the momentum direction.

The usual fermion-photon vertex is

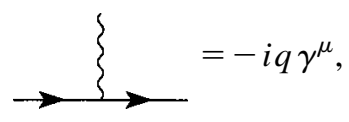

where $q$ is the fermion charge and $\mu$ is the space-time index on the photon line. The dimensionless coefficients for Lorentz and $C P T$ violation lead to the additional vertex

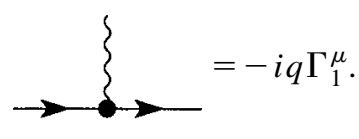


[1] See, for example, CPT and Lorentz Symmetry, edited by V. A. Kostelecký (World Scientific, Singapore, 1999).

[2] D. Colladay and V. A. Kostelecký, Phys. Rev. D 55, 6760 (1997); 58, 116002 (1998); Phys. Lett. B 511, 209 (2001).

[3] V. A. Kostelecký and R. Lehnert, Phys. Rev. D 63, 065008 (2001).

[4] R. Jackiw and V. A. Kostelecký, Phys. Rev. Lett. 82, 3572 (1999); M. Pérez-Victoria, J. High Energy Phys. 04, 032 (2001), and references therein.

[5] W. H. Furry, Phys. Rev. 81, 115 (1937).

[6] G. 't Hooft and M. Veltman, Nucl. Phys. B44, 189 (1972).

[7] C. G. Bollini and J. J. Giambiagi, Phys. Lett. 40B, 566 (1972); J. F. Ashmore, Lett. Nuovo Cim. 4, 289 (1972); G. M. Cicuta and E. Montaldi, ibid. 4, 329 (1972).

[8] W. Pauli and F. Villars, Rev. Mod. Phys. 21, 434 (1949).

[9] V. A. Kostelecký and S. Samuel, Phys. Rev. D 39, 683 (1989); 40, 1886 (1989); Phys. Rev. Lett. 63, 224 (1989); 66, 1811 (1991); V. A. Kostelecký and R. Potting, Nucl. Phys. B359, 545 (1991); Phys. Lett. B 381, 89 (1996); Phys. Rev. D 63, 046007 (2001); V. A. Kostelecký, M. Perry, and R. Potting, Phys. Rev. Lett. 84, 4541 (2000).

[10] S. M. Carroll et al., Phys. Rev. Lett. 87, 141601 (2001); Z. Guralnik et al., Phys. Lett. B 517, 450 (2001); A. Anisimov et al., hep-ph/0106356; C. E. Carlson et al., Phys. Lett. B 518, 201 (2001).

[11] KTeV Collaboration, Y. B. Hsiung et al., Nucl. Phys. B (Proc. Suppl.) 86, 312 (2000).

[12] OPAL Collaboration, R. Ackerstaff et al., Z. Phys. C 76, 401 (1997); DELPHI Collaboration, M. Feindt et al., preprint DELPHI 97-98 CONF 80, 1997; BELLE Collaboration, K. Abe et al., Phys. Rev. Lett. 86, 3228 (2001).

[13] V. A. Kostelecký and R. Potting, Phys. Rev. D 51, 3923 (1995); D. Colladay and V. A. Kostelecký, Phys. Lett. B 344, 259 (1995); Phys. Rev. D 52, 6224 (1995); V. A. Kostelecký and R. Van Kooten, ibid. 54, 5585 (1996); V. A. Kostelecký, Phys. Rev. Lett. 80, 1818 (1998); Phys. Rev. D 61, 016002
(2000); 64, 076001 (2001); N. Isgur et al., Phys. Lett. B 515, 333 (2001).

[14] O. Bertolami et al., Phys. Lett. B 395, 178 (1997).

[15] L. R. Hunter et al., in Ref. [1]; D. Bear et al., Phys. Rev. Lett. 85, 5038 (2000); D. F. Phillips et al., Phys. Rev. D 63, 111101(R) (2001); M. A. Humphrey et al., physics/0103068; V. A. Kostelecký and C. D. Lane, Phys. Rev. D 60, 116010 (1999); J. Math. Phys. 40, 6245 (1999); R. Bluhm et al., hep-ph/0111141.

[16] H. Dehmelt et al., Phys. Rev. Lett. 83, 4694 (1999); R. Mittleman et al., ibid. 83, 2116 (1999); G. Gabrielse et al., ibid. 82, 3198 (1999); R. Bluhm et al., ibid. 82, 2254 (1999); 79, 1432 (1997); Phys. Rev. D 57, 3932 (1998).

[17] B. Heckel et al., in Elementary Particles and Gravitation, edited by B. N. Kursunoglu et al. (Plenum, New York, 1999); R. Bluhm and V. A. Kostelecký, Phys. Rev. Lett. 84, 1381 (2000).

[18] S. M. Carroll, G. B. Field, and R. Jackiw, Phys. Rev. D 41, 1231 (1990); V. A. Kostelecký and M. Mewes, Phys. Rev. Lett. 87, 251304 (2001).

[19] V. W. Hughes et al., Phys. Rev. Lett. 87, 111804 (2001); R. Bluhm et al., ibid. 84, 1098 (2000).

[20] E. C. Stueckelberg and A. Petermann, Helv. Phys. Acta 26, 499 (1953); M. Gell-Mann and F. E. Low, Phys. Rev. 95, 1300 (1954); C. G. Callan, Phys. Rev. D 2, 1541 (1970); K. Symanzik, Commun. Math. Phys. 18, 227 (1970); K. G. Wilson and J. Kogut, Phys. Rep. 12, 75 (1974).

[21] See, for example, J. C. Collins, Renormalization (Cambridge University Press, Cambridge, England, 1986).

[22] G. 't Hooft, Nucl. Phys. B61, 455 (1973).

[23] For beta-function calculations in a general renormalizable gauge theory, see, for example, T. P. Cheng, E. Eichten, and L. F. Li, Phys. Rev. D 9, 2259 (1974); I. Jack and H. Osborn, J. Phys. A 16, 1101 (1983); M. E. Machacek and M. T. Vaughn, Nucl. Phys. B222, 83 (1983); A. G. M. Pickering, J. A. Gracey, and D. R. T. Jones, Phys. Lett. B 510, 347 (2001), and references therein. 